hep-ph/9412283

December 1994.

\title{
New Quark Distributions and Semi-Inclusive Electroproduction on Polarized Nucleons
}

\author{
Aram KOTZINIAN 円 \\ Yerevan Physics Institute, \\ Alikhanian Brothers St. 2; AM-375036 Yerevan, \\ Armenia.
}

\begin{abstract}
.
The quark-parton model calculation including the effects of intrinsic transverse momentum and of all six twist-two distribution functions of quarks in polarized nucleons is performed. It is demonstrated that new twist-two quark distribution functions and polarized quark fragmentation functions can be investigated in semi-inclusive DIS at leading order in $Q^{2}$. The general expression for the cross-section of semi-inclusive DIS of polarized leptons on polarized nucleons in terms of structure functions is also discussed.
\end{abstract}

\footnotetext{
${ }^{1}$ Now a visitor at CERN, PPE-Division, CH-1211, Geneva 23, Switzerland. E-mail:ARAM@CERNVM.CERN.CH
} 


\section{Introduction}

Deep-inelastic scattering (DIS) of leptons on nucleons provides an excellent tool for probing the structure of nuclear matter. The leading-twist momentum and helicity distribution functions (DF) of quarks in nucleons, $f_{1}(x)$ and $g_{1}(x)$, have been intensively studied. There exists another independent leading-twist DF, $h_{1}(x)$ [1], [2], describing the transverse-spin distribution of quarks in a transversely-polarized nucleon. These DF's depend on longitudinal momentum fraction $(x)$ carried by quark and are integrated over its intrinsic transverse momentum $\left(\vec{k}_{T}\right)$. In contrast to chirally even DF's $f_{1}(x)$ and $g_{1}(x)$, the chirally odd DF, $h_{1}(x)$, cannot be measured in simple DIS because in this case transverse spin asymmetries are suppressed at high energies. It can be measured in the lepton pair production process in nucleon-nucleon collisions with both nucleons polarized transversely. To be sensitive to the transversity distribution, it is necessary that either the sea quarks are highly polarized or that polarized antinucleons are used. Thus, at present this experiment seems very difficult.

In semi-inclusive DIS (SIDIS) the transverse-spin DF can be probed if the transverse polarization of the struck quark is measured in some way. This "quark polarimeter" may be provided by an azimuthal dependence of the fragmentation function ( $\mathrm{FF}$ ) for transversely-polarized quarks [3] (Collins effect). Another possibility of quark polarimetry is based on the observed correlation of flavor and electric charge of the fragmenting quark and leading hadron. In Ref. [4] it was proposed to measure the transverse polarization of quarks by measuring the polarization of self-analyzing baryons from fragmentation. An investigation of the transversity distribution, $h_{1}(x)$, in SIDIS on transversely-polarized nucleons has been proposed by the HELP collaboration [5].

Semi-inclusive DIS on a longitudinally-polarized target has been considered in Ref. [6] and [7]. It was proposed to measure asymmetries for production of different types of hadrons to get information about the flavor dependence of quark helicity DF in nucleons. This kind of measurements was already performed in the SMC experiment [8] and is planned by the HERMES collaboration 99.

In theoretical calculations of polarized SIDIS the intrinsic transverse momentum of the quarks in the nucleon is usually ignored. For example in 10 the polarized SIDIS cross section integrated over final hadron transverse momentum $\left(\vec{P}_{T}^{h}\right)$ was considered, keeping higher twist DF and FF. Because the intrinsic $k_{T}$ was neglected and the integration over $\vec{P}_{T}^{h}$ was assumed, the target transverse-spin asymmetry in this case appears only at twistthree $(\sim 1 / Q)$. But, as was shown in Ref. [3], the target transverse-spin asymmetry may exist at twist-two level in the azimuthal distribution of produced hadron. This asymmetry arises from the azimuthal dependence of transversely-polarized quark fragmentation and is sensitive to $h_{1}(x)$. The parton model picture of Ref. [3] is not symmetric in the sense that the transverse momentum of final hadron with respect to the scattered quark was taken into account but the intrinsic transverse momentum of the initial quark in nucleon was neglected.

It is known that even in unpolarized SIDIS the effect of intrinsic momentum can be significant [11, [12]. For a polarized nucleon the situation is more complicated. As was shown in Ref. [1] and [13] for the nonzero $k_{T}$ case, the quark distribution in a polarized nucleon is described by six DF's already at twist-two (instead of three DF's when intrinsic $k_{T}$ effects are neglected). The three "new" DF's relate the transverse 
(longitudinal) polarization of the quark to the longitudinal (transverse) polarization of nucleon. Measurement of these DF's was proposed in the doubly-polarized Drell-Yan process. Their contribution to polarized DIS appears only at twist-three $(\sim 1 / Q)$ [14].

The main subject of this article is the calculation of the polarized SIDIS cross section in the quark-parton model with nonzero intrinsic $k_{T}$. The lay-out of this paper is as follows: section 2 contains the derivation and discussion of a general expression for the polarized SIDIS cross section in terms of structure functions, section 3 contains a description of the quark-parton model with intrinsic $k_{T}$ for polarized SIDIS, in the section 4 a final expression for the SIDIS cross section is derived assuming exponential dependence on transverse momentum of DF's and FF's, section 5 contains a short discussion and illustrates possible ways of experimental investigation of the effects of different DF's and FF's in polarized SIDIS and finally section 6 contains some concluding remarks.

\section{Structure functions for polarized SIDIS}

Inelastic scattering of polarized leptons on polarized nucleons $l+N \rightarrow l^{\prime}+N^{\prime}+\pi$ was considered a long time ago by Dombey [15]. For polarized SIDIS, Gourdin [16] has counted the number of structure functions and derived some constraints on them from the requirement of positivity. To derive a general formula for the cross section these authors used the decomposition of the hadronic tensor into scalar structure functions corresponding to different polarizations of the virtual photon and target. I

In this section I follow the method used in [15] and [16] and present explicit formulae for polarized SIDIS cross-sections.

The Feynman diagram describing this process in the one-photon exchange approximation is depicted in Fig. 1.

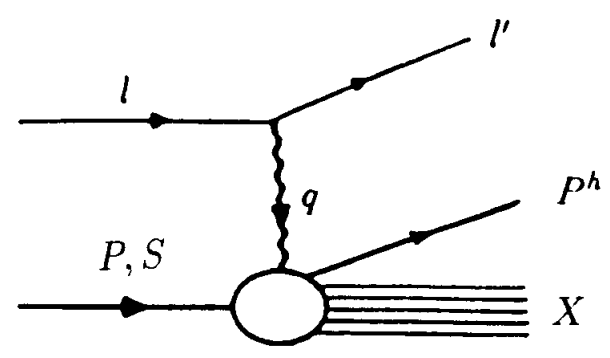

Figure 1: Lowest order diagram for SIDIS.

The standard notation for DIS variables is used: $l(E)$ and $l^{\prime}\left(E^{\prime}\right)$ are the momenta (energies) of the initial and final lepton; $\theta_{l}$ and $\phi_{l a b}^{l}$ are the scattered electron polar and azimuthal angles in the laboratory frame; $q=l-l^{\prime}$ is the exchanged virtual photon momentum; $\theta_{\gamma}$ is the virtual photon emission angle; $P$ is the target nucleon momentum; $P^{h}\left(E^{h}\right)$ is the final hadron momentum (energy); $Q^{2}=-q^{2}=4 E E^{\prime} / \sin ^{2}\left(\theta_{l} / 2\right) ; \nu=$ $P \cdot q / M ; x=Q^{2} / 2 P \cdot q ; y=\nu / E ; z=P \cdot P_{T}^{h} / P \cdot q$.

\footnotetext{
${ }^{2} \mathrm{~A}$ recent review on the subject of polarized lepton-nucleon scattering is given by S. Boffi, C.Giusti and F.D. Pacati [17]. The author thanks D. von Harrach for mentioning this article.
} 
For the cross-section one has

$$
d^{6} \sigma^{l+N \rightarrow l^{\prime}+h+X}=\frac{1}{4 P \cdot l}\left(\frac{4 \pi \alpha}{Q^{2}}\right)^{2} l_{\mu \nu} W^{\mu \nu}(2 \pi)^{4} \frac{d^{3} l^{\prime}}{(2 \pi)^{3} 2 E^{\prime}} \frac{d^{3} P^{h}}{(2 \pi)^{3} 2 E^{h}} .
$$

Here the leptonic tensor is given by QED:

$$
l_{\mu \nu}=2\left(l_{\mu} l_{\nu}^{\prime}+l_{\nu} l_{\nu}^{\prime}-l \cdot l^{\prime} g_{\mu \nu}+i \lambda \epsilon_{\mu \nu \alpha \beta} l^{\alpha} q^{\beta}\right)
$$

where $\lambda$ is the initial lepton helicity $(|\lambda| \leq 1)$.

The hadronic tensor in (1) is defined by

$$
W_{\mu \nu}=\sum_{X} \int \delta^{(4)}\left(P+q-P^{h}-P_{X}\right) \prod_{i \in X} \frac{d^{3} P_{i}}{(2 \pi)^{3} 2 E_{i}}\left\langle P^{h}, X\left|J_{\mu}\right| P, S\right\rangle\left\langle P, S\left|J_{\nu}\right| P^{h}, X\right\rangle,
$$

where $S$ is the target nucleon polarization.

The hadronic tensor can be decomposed into a spin-independent $\left(W_{\mu \nu}^{(0)}\right)$ and a spindependent $\left(W_{\mu \nu}^{(S)}\right)$ part:

$$
W_{\mu \nu}=W_{\mu \nu}^{(0)}+W_{\mu \nu}^{(S)}
$$

For a spin- $\frac{1}{2}$ target, $W_{\mu \nu}^{(S)}$ has to be linear in $S$ :

$$
W_{\mu \nu}^{(S)}=S^{\rho} W_{\mu \nu \rho}^{(S)}
$$

Note that, since $S$ is a pseudovector, $W_{\mu \nu \rho}$ is a pseudotensor.

It is convenient to consider the hadronic tensor decomposition in terms of structure functions in the target laboratory frame, with the $z$-axis chosen in the virtual photon momentum direction and the $x$-axis along the final hadron transverse momentum $\vec{P}_{T}^{h}$ (see Fig. 2). This reference system is referred to as the laboratory gamma-hadron frame $(\mathrm{LGHF})$.

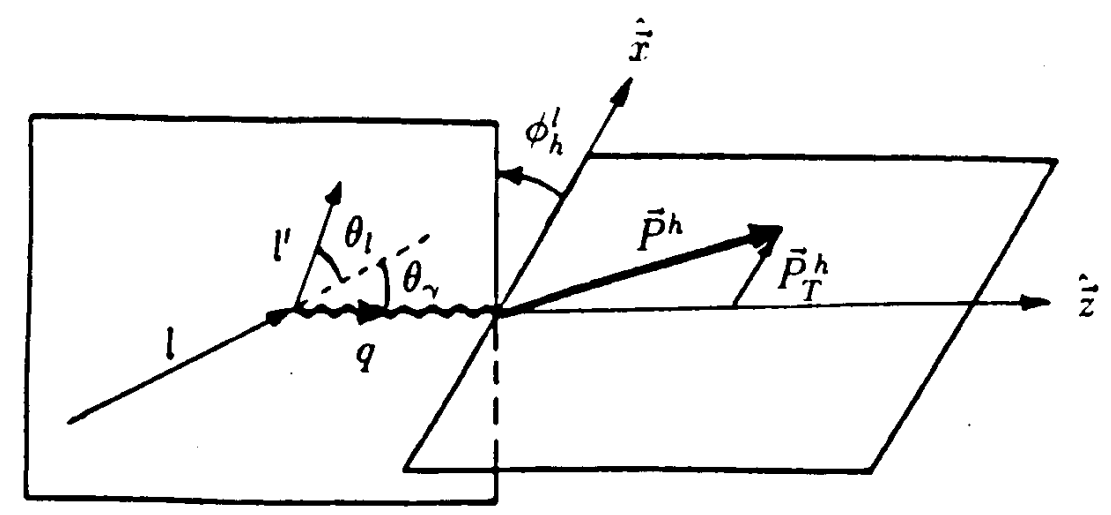

Figure 2: Lepton and produced hadron momenta in the LGHF.

To pass to this reference frame one has to perform three rotations:

1) Rotation around the initial lepton momentum by an angle $\phi_{l a b}^{l}$, to pass to the lepton scattering plane. 
2) Rotation around the normal to the lepton scattering plane by an angle $-\theta_{\gamma}$, defining a new $z$-axis coinciding with the virtual photon momentum direction. This often-used reference frame will be called the laboratory gamma-lepton frame (LGLF).

3) Rotation around the new $z$-axis by the azimuthal angle of the produced hadron $\left(\phi_{l}^{h}\right)$, defining a new $x$-axis coinciding with the produced hadron transverse momentum direction.

For the following it is important to note that, by definition, $\hat{\vec{z}}=\hat{\vec{z}}, \hat{\vec{x}}=\hat{\overrightarrow{P_{T}^{h}}}$ are polar vectors and $\hat{\vec{y}}=[\hat{\vec{z}} \times \hat{\vec{x}}]$ is an axial vector.

One of the advantages of the LGHF is that the hadronic tensor in this frame is independent of the relative azimuthal angle between the lepton and hadron planes $\left(\phi_{h}^{l}=-\phi_{l}^{h}\right)$. To decompose the hadronic tensor into scalar structure functions the following complete basis for polarization vectors of the virtual photon $\left(\epsilon^{\mu}\right)$ and nucleon $\left(e^{\mu}\right)$ is chosen in the laboratory frame: 向

$$
\begin{aligned}
\epsilon_{0}^{\mu} & =\frac{1}{Q}\left(q^{3}, 0,0, q^{0}\right), & e_{0}^{\mu} & =(1,0,0,0), \\
\epsilon_{1}^{\mu} & =(0,1,0,0), & e_{1}^{\mu} & =(0,1,0,0), \\
\epsilon_{2}^{\mu} & =(0,0,1,0), & e_{2}^{\mu} & =(0,0,1,0), \\
\epsilon_{3}^{\mu} & =\frac{q^{\mu}}{Q}, & e_{3}^{\mu} & =(0,0,0,1) .
\end{aligned}
$$

By construction, $\epsilon_{2}^{\mu}$ and $e_{2}^{\mu}$ are equal to $\hat{\vec{y}}$ and thus are axial vectors. The following closure properties hold for the basis vectors:

$$
\begin{aligned}
& g_{\mu \nu} \epsilon_{a}^{\mu} \epsilon_{b}^{\nu}=g_{a b}, \\
& g_{\mu \nu} e_{i}^{\mu} e_{j}^{\nu}=g_{i j}, \\
& g^{a b} \epsilon_{a}^{\mu} \epsilon_{b}^{\nu}=g^{\mu \nu}, \\
& g^{i j} e_{i}^{\mu} e_{j}^{\nu}=g^{\mu \nu} .
\end{aligned}
$$

Now, expanding both parts of the hadronic tensor over the complete basis of polarization vectors,

$$
\begin{aligned}
& W_{\mu \nu}^{(0)}=\epsilon_{\mu}^{a} \epsilon_{\nu}^{b} H_{a b}^{(0)}, \\
& W_{\mu \nu \rho}^{(S)}=\epsilon_{\mu}^{a} \epsilon_{\nu}^{b} e_{\rho}^{i} H_{a b i}^{(S)} .
\end{aligned}
$$

one has

$$
l_{\mu \nu} W^{\mu \nu}=L^{a b}\left(H_{a b}^{(0)}+S^{\rho} e_{\rho}^{i} H_{a b i}^{(S)}\right)
$$

where

$$
L^{a b}=\epsilon_{\mu}^{a} \epsilon_{\nu}^{b} l^{\mu \nu}
$$

can be treated as a virtual-photon polarization density matrix.

Using (7) one can express the scalar structure functions as

$$
\begin{aligned}
& H_{a b}^{(0)}=\epsilon_{a}^{\mu} \epsilon_{b}^{\nu} W_{\mu \nu}^{(0)}, \\
& H_{a b i}^{(S)}=\epsilon_{a}^{\mu} \epsilon_{b}^{\nu} e_{i}^{\rho} W_{\mu \nu \rho}^{(S)} .
\end{aligned}
$$

\footnotetext{
${ }^{3}$ Everywhere in this article unit vectors are denoted by a hat: $\hat{\vec{a}}=\vec{a} /|\vec{a}|$.

${ }^{4}$ Of course, this decomposition can be performed in a Lorentz invariant form.
} 
Let us consider the restrictions imposed by the invariance properties of $W_{\mu \nu}$ [16] :

- current conservation $\Rightarrow$ structure functions with $a=3$ or $b=3$ are equal to zero,

- parity conservation $\Rightarrow H_{a b}^{(0)}=0$ if it contains an odd number of indices 2 and $H_{a b i}^{(S)}=0$ if it contains an even number of indices 2 ,

- hermiticity of $W_{\mu \nu} \Rightarrow H_{a b}^{(0)}=H_{b a}^{(0) *}$ and $H_{a b i}^{(S)}=H_{b a i}^{(S) *}$,

- contribution of $H_{a b 0}^{(S)}$ to $W_{\mu \nu}$ is zero since $e_{0}^{\rho}=P^{\rho} / M$ and $S \cdot P=0$,

- time reversal invariance does not give new constraints on SIDIS structure functions.

Taking into account these properties one can choose the following set of independent real structure functions: five spin-independent $H_{00}^{(0)}, H_{11}^{(0)}, H_{22}^{(0)}, R e H_{01}^{(0)}, \operatorname{Im}_{01}^{(0)}$ and thirteen spin-dependent $H_{002}^{(S)}, R e H_{012}^{(S)}, I m H_{012}^{(S)}, e H_{021}^{(S)}, I m H_{021}^{(S)}, R e H_{023}^{(S)}, I m H_{023}^{(S)}, H_{112}^{(S)}$, $R e H_{121}^{(S)}, I m H_{121}^{(S)}, R e H_{123}^{(S)}, I m H_{123}^{(S)}, H_{222}^{(S)}$. $P_{T}^{h 2}$

In the general case these structure functions depend on four variables $Q^{2}, \nu, z$ and

The lepton momenta in the LGHF have the form:

$$
\begin{aligned}
& l_{\gamma h}^{\mu}=E\left(1, \sin \theta_{\gamma} \cos \phi_{l}^{h}, \sin \theta_{\gamma} \sin \phi_{l}^{h}, \cos \theta_{\gamma}\right), \\
& l_{\gamma h}^{\prime \mu}=E\left((1-y), \sin \theta_{\gamma} \cos \phi_{l}^{h}, \sin \theta_{\gamma} \sin \phi_{l}^{h},(1-y) \cos \left(\theta_{\gamma}+\theta_{l}\right)\right),
\end{aligned}
$$

with

$$
\begin{aligned}
\cos \theta_{\gamma} & =\sqrt{\frac{Q^{2}}{Q^{2}+4 M^{2} x^{2}}}\left(1+\frac{2 M^{2} x^{2} y}{Q^{2}}\right), \\
\sin \theta_{\gamma} & =\sqrt{\frac{4 M^{2} x^{2}}{Q^{2}+4 M^{2} x^{2}}\left(1-y-\frac{M^{2} x^{2} y^{2}}{Q^{2}}\right)} .
\end{aligned}
$$

By our choice of reference system the dependence on azimuthal angle $\phi_{h}^{l}$ is completely contained in the leptonic tensor. For $\mathcal{L}^{a b}=(1-\epsilon) L^{a b} / 2 Q^{2}$, one also has the hermiticity property $\mathcal{L}^{a b}=\mathcal{L}^{b a *}$ and from (2), (10) and (12) it follows that

$$
\begin{aligned}
\mathcal{L}^{00} & =\epsilon, \\
\mathcal{L}^{01} & =\sqrt{\frac{\epsilon(1+\epsilon)}{2}} \cos \phi_{h}^{l}+i \lambda \sqrt{\frac{\epsilon(1-\epsilon)}{2}} \sin \phi_{h}^{l}, \\
\mathcal{L}^{02} & =-\sqrt{\frac{\epsilon(1+\epsilon)}{2}} \sin \phi_{h}^{l}+i \lambda \sqrt{\frac{\epsilon(1-\epsilon)}{2}} \cos \phi_{h}^{l}, \\
\mathcal{L}^{11} & =\frac{1}{2}+\frac{\epsilon}{2} \cos 2 \phi_{h}^{l}, \\
\mathcal{L}^{12} & =-\frac{\epsilon}{2} \sin 2 \phi_{e}^{h}+i \frac{\lambda}{2} \sqrt{1-\epsilon^{2}} \\
\mathcal{L}^{22} & =\frac{1}{2}-\frac{\epsilon}{2} \cos 2 \phi_{h}^{l},
\end{aligned}
$$

where

$$
\epsilon=\frac{1}{1+2 \frac{\nu^{2}+Q^{2}}{Q^{2}} \operatorname{tg}^{2} \frac{\theta_{l}}{2}}=\frac{2(1-y)-M x y / E}{1+(1-y)^{2}+M x y / E}
$$

describes the virtual-photon polarization. 
One can see that all "memory" of the scattered lepton azimuthal angle, $\phi_{h}^{l}$, is contained in the polarization density matrix of virtual photon when SIDIS is described in the LGHF.

Finally, for the product of the hadronic and leptonic tensors we get

$$
\begin{aligned}
& \frac{1-\epsilon}{2 Q^{2}} l_{\mu \nu} W^{\mu \nu} \\
& =\frac{1}{2}\left(H_{11}^{(0)}+H_{22}^{(0)}\right)+\epsilon H_{00}^{(0)}+\sqrt{2 \epsilon(1+\epsilon)} \operatorname{Re} H_{01}^{(0)} \cos \phi_{h}^{l}+\frac{\epsilon}{2}\left(H_{11}^{(0)}-H_{22}^{(0)}\right) \cos 2 \phi_{h}^{l} \\
& +\lambda \sqrt{2 \epsilon(1-\epsilon)} \operatorname{Im} H_{01}^{(0)} \sin \phi_{h}^{l} \\
& -S_{(\gamma h)}^{1}\left[\sqrt{2 \epsilon(1+\epsilon)} \operatorname{Re} H_{021}^{(S)} \sin \phi_{h}^{l}+\epsilon \operatorname{Re} H_{121}^{(S)} \sin 2 \phi_{h}^{l}\right] \\
& -S_{(\gamma h)}^{2}\left[\frac{1}{2}\left(H_{112}^{(S)}+H_{222}^{(S)}\right)+\epsilon H_{002}^{(S)}+\sqrt{2 \epsilon(1+\epsilon)} R e H_{012}^{(S)} \cos \phi_{h}^{l}+\frac{\epsilon}{2}\left(H_{112}^{(S)}-H_{222}^{(S)}\right) \cos 2 \phi_{h}^{l}\right] \\
& -S_{(\gamma h)}^{3}\left[\sqrt{2 \epsilon(1+\epsilon)} \operatorname{Re} H_{023}^{(S)} \sin \phi_{h}^{l}+\epsilon R e H_{123}^{(S)} \sin 2 \phi_{h}^{l}\right] \\
& +\lambda S_{(\gamma h)}^{1}\left[-\sqrt{1-\epsilon^{2}} \operatorname{Im} H_{121}^{(S)}+\sqrt{2 \epsilon(1-\epsilon)} \operatorname{Im} H_{021}^{(S)} \cos \phi_{h}^{l}\right] \\
& -\lambda S_{(\gamma h)}^{2}\left[\sqrt{2 \epsilon(1-\epsilon)} \operatorname{Im} H_{012}^{(S)} \sin \phi_{h}^{l}\right] \\
& +\lambda S_{(\gamma h)}^{3}\left[-\sqrt{1-\epsilon^{2}} \operatorname{Im} H_{123}^{(S)}+\sqrt{2 \epsilon(1-\epsilon)} \operatorname{Im} H_{023}^{(S)} \cos \phi_{h}^{l}\right],
\end{aligned}
$$

where $S_{(\gamma h)}^{1}, S_{(\gamma h)}^{2}$ and $S_{(\gamma h)}^{3}$ are the target nucleon spin components in the LGHF. They are related to the target nucleon longitudinal $S_{\gamma l}^{L}$ and transverse $\vec{S}_{\gamma l}^{T}=\left(S_{\gamma l}^{1}, S_{\gamma l}^{2}\right)$ spin components in the LGLF by

$$
\begin{aligned}
& S_{\gamma h}^{1}=S_{\gamma l}^{T} \cos \left(\phi_{l}^{S}-\phi_{l}^{h}\right), \\
& S_{\gamma h}^{2}=S_{\gamma l}^{T} \sin \left(\phi_{l}^{S}-\phi_{l}^{h}\right), \\
& S_{\gamma h}^{3}=S_{\gamma l}^{L},
\end{aligned}
$$

and $\vec{S}_{\gamma l}$ is related to the target polarization in the laboratory frame by

$$
\begin{aligned}
& S_{\gamma l}^{1}=S_{l a b}^{T} \cos \theta_{\gamma} \cos \left(\phi_{l a b}^{S}-\phi_{l a b}^{l}\right)+S_{l a b}^{L} \sin \theta_{\gamma}, \\
& S_{\gamma l}^{2}=S_{l a b}^{T} \sin \left(\phi_{l a b}^{S}-\phi_{l a b}^{l}\right), \\
& S_{\gamma l}^{3}=-S_{l a b}^{T} \sin \theta_{\gamma} \cos \left(\phi_{l a b}^{S}-\phi_{l a b}^{l}\right)+S_{l a b}^{L} \cos \theta_{\gamma},
\end{aligned}
$$

where $S_{l a b}^{L}$ and $\vec{S}_{l a b}^{T}$ are the nucleon spin longitudinal and transverse components with respect to initial lepton momentum.

Note that in (16)-(18) the dependence of the polarized SIDIS cross section on target spin components and produced hadron azimuthal angle is expressed in explicit form. In principle, it is possible to separate the different structure functions contribution by using a "Fourier analysis" on $\phi_{l}^{h}$ for different beam and target polarizations as was done for the unpolarized case in Ref. [12].

Let us now consider the cross section expression in the LGLF

$$
\frac{d^{6} \sigma^{l+N \rightarrow l^{\prime}+h+X}}{d x d y d \phi_{l a b}^{l} d z d P_{T}^{h 2} d \phi_{l}^{h}}=\frac{\nu}{4 P_{\|}^{h}} \frac{\alpha^{2} y}{2 Q^{4}} l_{\mu \nu} W^{\mu \nu},
$$


and integrate it over $\phi_{l}^{h}$. One gets

$$
\begin{aligned}
& \int_{0}^{2 \pi} d \phi_{l}^{h} \frac{d^{6} \sigma^{l+N \rightarrow l^{\prime}+h+X}}{d x d y d \phi_{l a b}^{l} d z d P_{T}^{h 2} d \phi_{l}^{h}}=\frac{\nu}{4 P_{\|}^{h}} \frac{2 \pi \alpha^{2} y}{Q^{2}(1-\epsilon)}\left[\frac{1}{2}\left(H_{11}^{(0)}+H_{22}^{(0)}\right)+\epsilon H_{00}^{(0)}\right. \\
& +S_{(\gamma l)}^{2} \sqrt{\frac{\epsilon(1+\epsilon)}{2}}\left(R e H_{021}^{(S)}-R e H_{012}^{(S)}\right) \\
& \left.+\lambda S_{(\gamma l)}^{1} \sqrt{\frac{\epsilon(1-\epsilon)}{2}}\left(I m H_{021}^{(S)}-I m H_{012}^{(S)}\right)-\lambda S_{(\gamma l)}^{3} \sqrt{1-\epsilon^{2}} I m H_{123}^{(S)}\right]
\end{aligned}
$$

It is interesting to note that, due to the presence of the third term in the rhs of the last equation, when integrated over $\phi_{l}^{h}$, the cross section can still have a single target-spin asymmetry. Furthermore integration over $P_{T}^{h 2}$ and $z$ has to give us the polarized DIS cross section times the mean hadron multiplicity $\left(\left\langle n_{h}\left(x, Q^{2}\right)\right\rangle\right)$.

One can show that by integrating over the produced hadron phase space, the first and last two terms in (22) precisely reproduce the exact formula for the polarized DIS cross section, given for example in Ref. [18], and that the following kinematical sum rules hold:

$$
\begin{aligned}
& \int \frac{d^{3} P^{h}}{2 E^{h}}\left[\frac{1}{2}\left(H_{11}^{(0)}+H_{22}^{(0)}\right)\right]=\left\langle n_{h}\left(x, Q^{2}\right)\right\rangle F_{1}^{D I S}\left(x, Q^{2}\right), \\
& \int \frac{d^{3} P^{h}}{2 E^{h}}\left[\frac{1}{2}\left(H_{11}^{(0)}+H_{22}^{(0)}\right)+H_{00}^{(0)}\right] \frac{2 x Q^{2}}{Q^{2}+4 M^{2} x^{2}}=\left\langle n_{h}\left(x, Q^{2}\right)\right\rangle F_{2}^{D I S}\left(x, Q^{2}\right), \\
& \int \frac{d^{3} P^{h}}{2 E^{h}}\left[\frac{2 M x}{Q}\left(I m H_{021}^{(S)}-\operatorname{Im}_{012}^{(S)}\right)-2 I m H_{123}^{(S)}\right]=\left\langle n_{h}\left(x, Q^{2}\right)\right\rangle g_{1}^{D I S}\left(x, Q^{2}\right), \\
& \int \frac{d^{3} P^{h}}{2 E^{h}}\left[\frac{Q}{2 M x}\left(I m H_{021}^{(S)}-I m H_{012}^{(S)}\right)+2 \operatorname{Im} H_{123}^{(S)}\right]=\left\langle n_{h}\left(x, Q^{2}\right)\right\rangle g_{2}^{D I S}\left(x, Q^{2}\right),
\end{aligned}
$$

where $F_{1}^{D I S}\left(x, Q^{2}\right), F_{2}^{D I S}\left(x, Q^{2}\right), g_{1}^{D I S}\left(x, Q^{2}\right)$ and $g_{2}^{D I S}\left(x, Q^{2}\right)$ are the structure functions entering in the spin-independent and spin-dependent part of the polarized DIS cross section.

As is well known, a single target-spin asymmetry is forbidden in simple DIS by time reversal invariance. This means that the following "sum rule" holds:

$$
\int \frac{d^{3} P^{h}}{2 E^{h}}\left(R e H_{021}^{(S)}-R e H_{012}^{(S)}\right)=0
$$

Thus, one can have a single target-spin asymmetry in a SIDIS cross section integrated over $\phi_{l}^{h}$, which disappears after integration over $P_{T}^{h 2}$ and $z$. This observation will be confirmed in the simple quark-parton model.

\section{Parton model with intrinsic transverse momentum}

Unpolarized SIDIS is described in a simple way by the factorized parton model. In this model the lepton knocks out a quark, which subsequently fragments into hadrons. Sixteen years ago it was shown by Cahn [11] that nonperturbative effects of the intrinsic transverse momentum $\left(k_{T}\right)$ of the quarks inside the nucleon may induce significant hadron 
asymmetries in the relative azimuthal angle $\phi_{l}^{h}$. The EMC experiment [12] found an azimuthal asymmetry at the level of up to $15-20 \%$, which arises mainly from the effects of the intrinsic $k_{T}$ of the struck quark with $\left\langle k_{T}^{2}\right\rangle \geq(0.44 \mathrm{GeV})^{2}$.

Here I generalize the calculation of Ref. [11] to the polarized SIDIS process. Calculations will be performed in the electron-quark scattering Breit-frame (BF) (Fig. 3), which can be reached from the LGLF by a Lorentz boost along the $z$-axis.

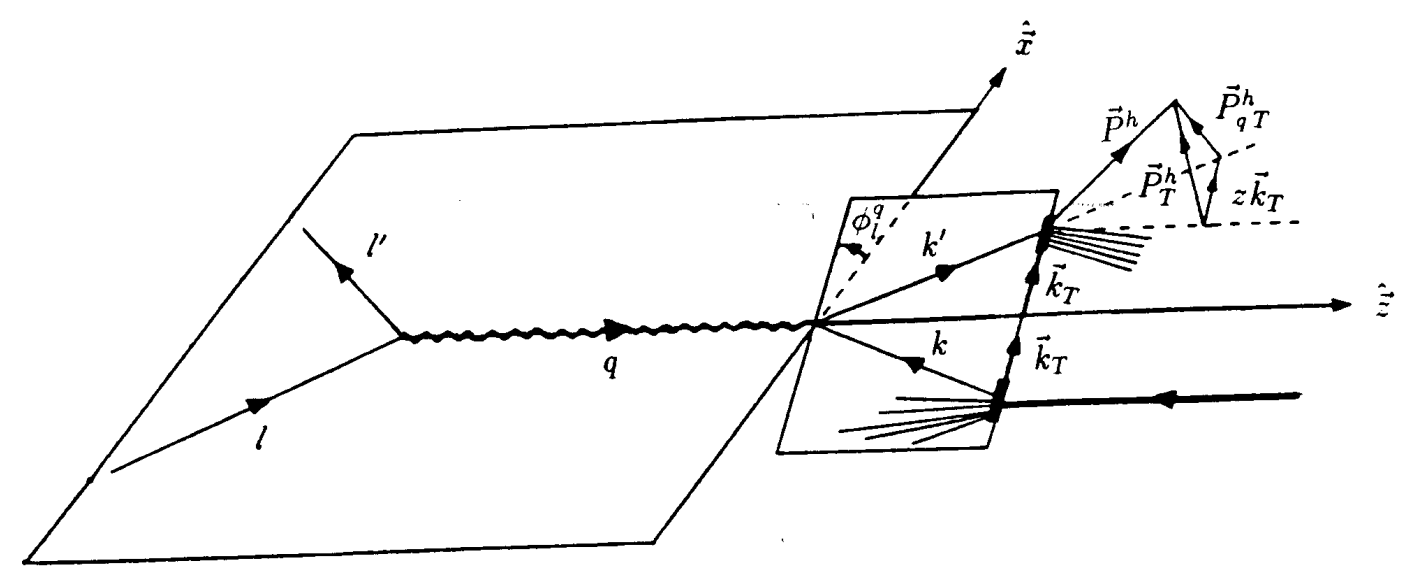

Figure 3: The quark-parton model picture for SIDIS in the BF.

The azimuthal angle, $\phi_{e}^{h}$, remains unchanged under this transformation, and, for the virtual photon four-momentum, we have $q^{\mu}=(0,0,0, Q)$. In the following calculations the terms $\sim 1 / Q^{2}$ are neglected. In this approximation the lepton and quark $\left(k, k^{\prime}\right)$ four-momenta in the $\mathrm{BF}$ are given by:

$$
\begin{aligned}
l_{B r}^{\mu} & =\frac{Q}{2}\left(\frac{2-y}{y}, \frac{2 \sqrt{1-y}}{y} \hat{\vec{l}}_{T}, 1\right), \\
l_{B r}^{\prime \mu} & =\frac{Q}{2}\left(\frac{2-y}{y}, \frac{2 \sqrt{1-y}}{y} \hat{\vec{l}}_{T},-1\right), \\
k_{B r}^{\mu} & =\frac{Q}{2}\left(1, \frac{2 \vec{k}_{T}}{Q},-1\right), \\
k_{B r}^{\prime \mu} & =\frac{Q}{2}\left(1, \frac{2 \vec{k}_{T}}{Q}, 1\right),
\end{aligned}
$$

where $\hat{\vec{l}}_{T}=(1,0)$ is the unit transverse two-vector in the lepton transverse momentum direction. Note that because of a nonzero $\vec{k}_{T}$, the electron and the quark scattering planes do not coincide in general (see Fig. 3, where the angle between these planes is denoted by $\phi_{l}^{q}$ ).

In the quark-parton model the description of the SIDIS process is similar to that of double scattering experiments, in which the polarization of the particle produced after the first scattering is measured. To calculate the cross section one has to know:

1) the description of the initial quark state,

2) the noncoplanar polarized $l+q \rightarrow l^{\prime}+q^{\prime}$ scattering cross section,

3) the description of the polarized quark fragmentation. 


\subsection{The initial quark state.}

The polarization of free quarks is most clearly described by the density matrix of partially polarized fermions, which in the ultrarelativistic case takes the form [19

$$
\rho=\frac{1}{2}(\gamma \cdot k)\left[1+\gamma^{5} s_{L}+\gamma^{5} \vec{\gamma}_{T} \cdot \vec{s}_{T}\right]
$$

where $s_{L}$ and $\vec{s}_{T}$ are the longitudinal and transverse (with respect to quark three-momentum $\vec{k})$ components of twice the quark polarization vector in its rest frame, $s_{L}^{2}+\left|\vec{s}_{T}\right|^{2} \leq 1$.

For the initial quark state in the nucleon one has to use, instead of (2), the following expression:

$$
\rho_{N}^{q(i n)}=\frac{1}{2} \mathcal{P}_{N}^{q}\left(x, k_{T}^{2}\right)(\gamma \cdot k)\left[1+\gamma^{5} s_{L}^{(i n)}\left(x, \vec{k}_{T}\right)+\gamma^{5} \vec{\gamma}_{T} \cdot \vec{s}_{T}^{(i n)}\left(x, \vec{k}_{T}\right)\right]
$$

where $\mathcal{P}_{N}^{q}\left(x, k_{T}^{2}\right), s_{L}^{(i n)}\left(x, \vec{k}_{T}\right)$ and $\vec{s}_{T}^{(i n)}\left(x, \vec{k}_{T}\right)$ are the probability, longitudinal and transverse polarization distributions. They can easily be found from the density matrix by calculating the appropriate trace

$$
\begin{aligned}
& \mathcal{P}_{N}^{q}\left(x, k_{T}^{2}\right)=\frac{1}{2 p^{0}} \operatorname{tr}\left(\rho_{N}^{q(i n)} \gamma^{0}\right), \\
& \mathcal{P}_{N}^{q}\left(x, k_{T}^{2}\right) s_{L}^{(i n)}\left(x, \vec{k}_{T}\right)=\frac{1}{2 p^{0}} \operatorname{tr}\left(\rho_{N}^{q(i n)} \gamma^{5} \gamma^{0}\right), \\
& \mathcal{P}_{N}^{q}\left(x, k_{T}^{2}\right) \vec{s}_{T}^{(i n)}\left(x, \vec{k}_{T}\right)=\frac{1}{2 p^{0}} \operatorname{tr}\left(\rho_{N}^{q(i n)} \gamma^{5} \vec{\gamma}_{T} \gamma^{0}\right) .
\end{aligned}
$$

At first sight, it seems that presence of the intrinsic transverse momentum of the quark can not give a sizable effect on the spin distribution. Consider, for example, a transversely-polarized quark in the quark-parton model. In this case the projection of quark polarization vector onto the nucleon momentum direction is proportional to $k_{T} / k_{\|}=$ $2 k_{T} / Q$, and one might conclude that the contribution of the quark transverse polarization to the nucleon longitudinal polarization is suppressed at high $Q^{2}$. However, the nucleon polarization has to be calculated in its rest frame, and the factor $1 / Q$ will disappear after a Lorentz transformation to nucleon rest frame.

Consider a simple example for spin transfer from a polarized nucleon to a quark. Let $A_{\mu}$ and $a_{\mu}$ be the polarization four-vectors of the nucleon and the quark in the frame where the nucleon has large momentum (for example in the BF), and suppose that they are related by $a_{0}=\alpha A_{0}, a_{3}=\alpha A_{3}, a_{1}=\beta A_{1}$ and $a_{2}=\beta A_{2}$, with $A_{0}=P_{N} S_{L} / M$, $A_{3}=E_{N} S_{L} / M, \vec{A}_{T}=\vec{S}_{T}$, where $\vec{S}$ is the nucleon polarization in its rest frame and $P_{N}$ $\left(E_{N}\right)$ is the momentum (energy) of the nucleon in the BF. Now one can calculate the quark polarization in its rest frame $(\vec{s})$ assuming that $m_{q}=x M$. After rotation of the coordinate system of the BF and a Lorentz boost along the quark momentum one gets: $s_{L}=\alpha S_{L}+\beta \vec{k}_{T} \cdot \vec{S}_{T} / m_{q}$ and $\vec{s}_{T}=\beta \vec{S}_{T}-\alpha \vec{k}_{T} S_{L} / m_{q}$. Thus, in this "toy" parton model, the longitudinal spin of the quark receives two contributions: from both the longitudinal and transverse spin of the nucleon. It is important to note that neither of these contributions is suppressed at high $Q^{2}$. The same behavior is true for the transverse spin of the quark. 
General consideration of the quark DF in a polarized nucleon in the case of nonvanishing $k_{T}$ has been done by Ralston and Soper [四] and recently by Tangerman and Mulders [13]. They have found that at the leading twist one needs six independent DF's depending on $x$ and $k_{T}^{2}$ : $f, g_{1 L}, g_{1 T}, h_{1 T}, h_{1 L}^{\perp}$, and $h_{1 T}^{\perp}$. The distributions $s_{L}^{(i n)}\left(x, \vec{k}_{T}\right)$ and $\vec{s}_{T}^{(i n)}\left(x, \vec{k}_{T}\right)$ are given by 13

$$
\begin{aligned}
& \mathcal{P}_{N}^{q}\left(x, k_{T}^{2}\right)=f\left(x, k_{T}^{2}\right), \\
& \mathcal{P}_{N}^{q}\left(x, k_{T}^{2}\right) s_{L}^{(i n)}\left(x, \vec{k}_{T}\right)=g_{1 L}\left(x, k_{T}^{2}\right) S_{L}+g_{1 T}\left(x, k_{T}^{2}\right) \frac{\vec{k}_{T} \cdot \vec{S}_{T}}{m_{D}}, \\
& \mathcal{P}_{N}^{q}\left(x, k_{T}^{2}\right) \vec{s}_{T}^{(i n)}\left(x, \vec{k}_{T}\right)=h_{1 T}\left(x, k_{T}^{2}\right) \vec{S}_{T}+\left[h_{1 L}^{\perp}\left(x, k_{T}^{2}\right) S_{L}+h_{1 T}^{\perp}\left(x, k_{T}^{2}\right) \frac{\vec{k}_{T} \cdot \vec{S}_{T}}{m_{D}}\right] \frac{\vec{k}_{T}}{m_{D}},
\end{aligned}
$$

where $m_{D}$ is an unknown mass parameter, $S_{L}$ and $\vec{S}_{T}$ are the nucleon longitudinal and transverse polarization with respect to its momentum. The "new" DF's have clear physical interpretation: for example, $g_{1 T}$ describes the quark longitudinal polarization in a transversely-polarized nucleon. It is important to notice that due to this DF even the initial quark longitudinal spin distribution in a polarized nucleon exhibits an azimuthal asymmetry.

\subsection{Noncoplanar polarized $l+q \rightarrow l^{\prime}+q^{\prime}$ scattering}

Using standard methods of QED [19], it is easy to calculate the cross section of a polarized lepton scattering on a polarized quark in the one-photon approximation:

$$
\begin{aligned}
\frac{d^{2} \sigma_{i}^{l+q \rightarrow l^{\prime}+q^{\prime}}}{d y d \phi_{h}^{l}}= & \frac{\alpha^{2} e_{i}^{2} y}{2 Q^{2}} f\left(x, k_{T}^{2}\right)\left[\left(s^{2}+u^{2}\right)\left(1+s_{L}^{(i n)} s_{L}^{\prime}\right)+\left(s^{2}-u^{2}\right) \lambda\left(s_{L}^{(i n)}+s_{L}^{\prime}\right)\right. \\
& \left.+2 s u\left(s_{T}^{(i n)} \cdot s_{T}^{\prime}\right)-4 u\left(s_{T}^{(i n)} \cdot l\right)\left(s_{T}^{\prime} \cdot l^{\prime}\right)-4 s\left(s_{T}^{(i n)} \cdot l^{\prime}\right)\left(s_{T}^{\prime} \cdot l\right)\right] .
\end{aligned}
$$

Here, $e_{i}$ is the electric charge of the quark in positron charge units, $s_{L}^{\prime}$ is the parameter describing the longitudinal spin component of the final quark and $s_{T}^{\prime}$ is four-vector describing its transverse spin; $s, t$ and $u$ are the usual Mandelstam variables given by

$$
\begin{aligned}
& s=2 M E x\left[1-2 \sqrt{1-y} \frac{\vec{k}_{T} \cdot \hat{\vec{l}}_{T}}{Q}\right] \\
& t=-Q^{2}=-2 M E x y \\
& u=-2 M E x(1-y)\left[1-\frac{2}{\sqrt{1-y}} \frac{\vec{k}_{T} \cdot \hat{\vec{l}}_{T}}{Q}\right] .
\end{aligned}
$$

Note that for noncoplanar $l+q \rightarrow l^{\prime}+q^{\prime}$ scattering $s$ and $u$ depend on the relative azimuthal angle between the quark and lepton scattering planes, $\phi_{l}^{q}$, and kinematical corrections of order $1 / Q$ will arise due to this dependence. 
The transverse polarization four-vectors of the quarks in the BF in this approximation are

$$
\begin{aligned}
& s_{T}^{(i n) \mu}=\left(0, \vec{s}_{T}^{(i n)}, \frac{2}{Q} \vec{k}_{T} \cdot \vec{s}_{T}^{(i n)}\right), \\
& s_{T}^{\prime \mu}=\left(0, \vec{s}_{T}^{\prime},-\frac{2}{Q} \vec{k}_{T} \cdot \vec{s}_{T}^{\prime}\right) .
\end{aligned}
$$

Substituting (29) and (30) into (28) the following expression for the cross section is obtained:

$$
\frac{d^{2} \sigma_{i}^{l+q \rightarrow l^{\prime}+q^{\prime}}}{d y d \phi_{h}^{l}}=\frac{\alpha^{2} e_{i}^{2}}{2 Q^{2} y} f\left(x, k_{T}^{2}\right)\left(a+b_{L} s_{L}^{\prime}+\vec{b}_{T} \cdot \vec{s}_{T}^{\prime}\right),
$$

where

$$
\begin{aligned}
a= & +(1-y)^{2}-4(2-y) \frac{\vec{k}_{T} \cdot \hat{\vec{l}}_{T}}{Q}+\lambda s_{L}^{(i n)} y\left[2-y-4 \sqrt{1-y} \frac{\vec{k}_{T} \cdot \hat{\vec{l}}_{T}}{Q}\right], \\
b_{L}= & \left.\lambda y\left[2-y-4 \sqrt{1-y} \frac{\vec{k}_{T} \cdot \hat{\vec{l}}_{T}}{Q}\right]+s_{L}^{(i n)}\right)\left[1+(1-y)^{2}-4(2-y) \sqrt{1-y} \frac{\vec{k}_{T} \cdot \hat{\vec{l}}_{T}}{Q}\right], \\
\vec{b}_{T}= & 2(1-y)\left\{\vec{s}_{T}^{(i n)}-2\left(\vec{s}_{T}^{(i n)} \cdot \hat{\vec{l}}_{T}\right) \hat{\vec{l}}_{T}\right. \\
& \left.+2 \frac{2-y}{Q \sqrt{1-y}}\left[\left(\vec{s}_{T}^{(i n)} \cdot \hat{\vec{l}}_{T}\right) \vec{k}_{T}+\left(\vec{s}_{T}^{(i n)} \cdot \vec{k}_{T}\right) \hat{\vec{l}}_{T}-\left(\hat{\vec{l}}_{T} \cdot \vec{k}_{T}\right) \vec{s}_{T}^{(i n)}\right]\right\} .
\end{aligned}
$$

The final quark polarization according to the general rules (see [19],§65) is given by

$$
\begin{aligned}
& s_{L}^{(f)}=\frac{b_{L}}{a}, \\
& \vec{s}_{T}^{(f)}=\frac{\vec{b}_{T}}{a} .
\end{aligned}
$$

From (32) and (33) one can see that the final quark can be transversely polarized only if the transverse polarization of the initial quark is not equal to zero. The sideways $\left(D_{s s}\right)$ and normal $\left(D_{n n}\right)$ transverse spin transfer coefficients to leading order in $1 / Q$ are given by

$$
D_{n n}=-D_{s s}=\frac{2(1-y)}{1+(1-y)^{2}+\lambda s_{L}^{(i n)} y(2-y)} .
$$

The azimuthal angle of the final quark transverse spin $\left(\phi_{l}^{\prime} S\right)$ in this approximation is very simply connected with that of the initial quark $\left(\phi_{l}^{S}\right)$ :

$$
\phi_{l}^{\prime} S=\pi-\phi_{l}^{S}
$$

For unpolarized leptons, expression (34) coincides with the depolarization factor in Ref. [3].

Note also that, in contrast with transverse polarization, the longitudinal polarization of the final quark is not equal to zero even if the initial quark is unpolarized but the initial lepton is longitudinally polarized. 
For the final quark state before fragmentation, one can now write the density matrix as

$$
\rho^{q(f)}=\frac{\alpha^{2} e_{i}^{2}}{2 Q^{2} y} f\left(x, k_{T}^{2}\right) a\left(1+\gamma^{5} s_{L}^{(f)}+\gamma^{5} \vec{\gamma}_{T} \cdot \vec{s}_{T}^{(f)}\right) .
$$

One can see that the final quark state has an azimuthal asymmetry in the relative angle between the lepton and quark scattering planes, $\phi_{l}^{q}$. Part of this asymmetry has a kinematical origin, as the third term in the expression for $a$ in (32). The azimuthal asymmetry in unpolarized SIDIS arises from this term [11]. But there also exist terms reflecting the azimuthal anglular dependence of the initial quark distributions, which are not suppressed at high $Q^{2}$.

\subsection{Polarized quark fragmentation.}

In analogy with the quark probability distribution in the nucleon one can write the probability of producing a hadron, $h$, in the polarized quark fragmentation as

$$
\mathcal{P}_{q}^{h}\left(z, \vec{P}_{q T}^{h}\right)=\frac{1}{2 p^{0}} \operatorname{tr}\left(\rho^{q(f)} F\left(z, \vec{P}_{q T}^{h}\right) \gamma^{0}\right)
$$

where $F\left(z, \vec{P}_{q T}^{h}\right)$ is the polarized quark fragmentation function, depending on $z=E^{h} / E^{q^{\prime}}$ and the hadron transverse momentum with respect to the final quark momentum $\vec{P}_{q T}^{h}=$ $\vec{P}_{T}^{h}-z \vec{k}_{T}$. This FF can be presented as a sum of spin-independent and spin-dependent parts [3].

$$
F\left(z, \vec{P}_{q T}^{h}\right)=F^{(0)}\left(z, P_{q T}^{h 2}\right)+\gamma^{5}\left(\vec{\gamma}_{T} \cdot\left[\hat{\overrightarrow{k^{\prime}}} \times \frac{\vec{P}_{q T}^{h}}{m_{F}}\right]\right) F^{(S)}\left(z, P_{q T}^{h 2}\right),
$$

where $m_{F}$ is another unknown mass parameter. The two terms on the rhs of equation (40) are the only ones allowed by parity invariance.

Calculating the trace in (37) one gets

$$
\begin{aligned}
\mathcal{P}_{q}^{h}\left(z, \vec{P}_{q T}^{h}\right) & =\frac{\alpha^{2} e_{i}^{2}}{2 Q^{2} y} f\left(x, k_{T}^{2}\right)\left(a F^{(0)}\left(z, P_{q T}^{h 2}\right)-\left(\vec{b}_{T} \cdot\left[\hat{\vec{k}}^{\prime} \times \frac{\vec{P}_{q T}^{h}}{m_{F}}\right]\right) F^{(S)}\left(z, P_{q T}^{h 2}\right)\right) \\
& =\frac{\alpha^{2} e_{i}^{2}}{2 Q^{2} y} f\left(x, k_{T}^{2}\right)\left(a F^{(0)}\left(z, P_{q T}^{h 2}\right)+\left|\vec{b}_{T}\right| \frac{\left|\vec{P}_{q T}^{h}\right|}{m_{F}} F^{(S)}\left(z, P_{q T}^{h 2}\right) \sin \phi_{c o l}\right)
\end{aligned}
$$

where $\phi_{c o l}$ is the angle between $\vec{P}_{q T}^{h}$ and the final quark transverse polarization.

In contrast to the ordinary $\mathrm{FF}, F^{(0)}\left(z, P_{T}^{2}\right)$, the spin-dependent part of the polarized quark FF, $F^{(S)}\left(z, P_{T}^{2}\right)$, or, in other words, the analyzing power of polarized fragmentation, has never been measured.

\section{Results}

In this section the polarized SIDIS cross section is calculated taking into account all six twist-two quark DF's and the Collins effect in polarized quark fragmentation. Kinematical corrections of order $1 / Q$ also are kept. 
To calculate the cross section one has to integrate over $\vec{k}_{T}$ and sum over all quark and antiquark types the probability of hadron production in final quark fragmentation (the initial quark probability distribution and $l+q \rightarrow l^{\prime}+q^{\prime}$ scattering cross section is already included in the final quark density matrix):

$$
\frac{d^{6} \sigma^{l+N \rightarrow l^{\prime}+h+X}}{d x d y d \phi_{l a b}^{l} d z d P_{T}^{h 2} d \phi_{l}^{h}}=\sum_{q} \int d^{2} k_{T} \mathcal{P}_{q}^{h}\left(z, \vec{P}_{q T}^{h}\right),
$$

where $d^{2} k_{T}=1 / 2 d k_{T}^{2} d \phi_{l}^{q}$ and $\phi_{l}^{q}$ is the quark azimuthal angle in the BF.

This integration can be performed analytically if one supposes that the transverse momentum dependence in the DF's and FF's may be written in factorized exponential form:

$$
\begin{aligned}
& d_{J}\left(x, k_{T}^{2}\right)=\frac{1}{\pi a_{J}} \exp \left(-\frac{k_{T}^{2}}{a_{J}}\right) d_{J}(x), \\
& F^{(0, S)}\left(z, p_{T}^{2}\right)=\frac{1}{\pi a_{F}^{(0, S)}} \exp \left(-\frac{p_{T}^{2}}{a_{F}^{(0, S)}}\right) F^{(0, S)}(z) .
\end{aligned}
$$

Here the index $J=f_{1}, g_{1 L}, g_{1 T}, h_{1 T}, h_{1 L}^{\perp}, h_{1 T}^{\perp}$ enumerates the different DF's. The width of transverse momentum distribution for each distribution (fragmentation) function $a_{J} \sim$ $\left\langle k_{T}^{2}\right\rangle\left(a_{F}^{(0, S)} \sim\left\langle P_{q T}^{h 2}\right\rangle\right)$ can in principle depend on $x(z)$.

After some calculations the final result looks like:

$$
\frac{d^{6} \sigma^{l+N \rightarrow l^{\prime}+h+X}}{d x d y d \phi_{l a b}^{l} d z d P_{T}^{h 2} d \phi_{l}^{h}}=\sum_{q} \frac{\alpha^{2} e_{q}^{2}}{2 Q^{2} y}\left(C_{U P}+C_{D P}+C_{S P}\right)
$$

where the contributions to unpolarized, double (beam and target) and single (target only) polarized parts of the cross section are given in the following three formulae.

$$
\begin{gathered}
C_{U P}=A_{f_{1}}^{(0)}\left[1+(1-y)^{2}-4 y(2-y) \frac{\alpha_{f_{1}}^{(0)} P_{T}^{h}}{Q} \cos \phi_{l}^{h}\right], \\
C_{D P}=\lambda S_{\gamma l}^{L} A_{g_{1 L}}^{(0)} y\left[2-y-4 \sqrt{1-y} \frac{\alpha_{g_{1 L}}^{(0)} P_{T}^{h}}{Q} \cos \phi_{l}^{h}\right] \\
+\lambda S_{\gamma l}^{T} A_{g_{1 T}}^{(0)} y\left[\left(2-y-4 \sqrt{1-y} \frac{\alpha_{g_{1 T}}^{(0)} P_{T}^{h}}{Q} \cos \phi_{l}^{h}\right) \frac{\alpha_{g_{1 T}}^{(0)} P_{T}^{h}}{m_{D}} \cos \left(\phi_{l}^{h}-\phi_{l}^{S}\right)\right. \\
\left.-2 \sqrt{1-y} \frac{b_{g_{1 T}}^{(0)}}{m_{D} Q} \cos \left(\phi_{l}^{S}\right)\right], \\
C_{S P}=-2 \frac{1-y}{m_{F}}\left\{S_{\gamma l}^{T} A_{h_{1 T}}^{(S)}\left(1-z \alpha_{h_{1 T}}^{(S)}\right) P_{T}^{h} \sin \left(\phi_{l}^{h}+\phi_{l}^{S}\right)\right. \\
+S_{\gamma l}^{L} \frac{A_{h_{1 L}^{\perp}}^{(S)} \alpha_{h_{1 L}^{\perp}}^{(S)}\left(1-z \alpha_{h_{1 L}^{\perp}}^{(S)}\right) P_{T}^{h 2} \sin 2 \phi_{l}^{h}}{m_{D}}
\end{gathered}
$$




$$
\begin{gathered}
+S_{\gamma l}^{T} \frac{A_{h_{1 T}^{\perp}}^{(S)} P_{T}^{h}\left(\alpha_{h_{1 T}^{\perp}}^{(S) 2}\left(1-z \alpha_{h_{1 T}^{\perp}}^{(S)}\right) P_{T}^{h 2} \cos \left(\phi_{l}^{h}-\phi_{l}^{S}\right) \sin 2 \phi_{l}^{h}\right.}{m_{D}^{2}} \\
\left.+\left(\frac{1}{2}-z \alpha_{h_{1 T}^{\perp}}^{(S)}\right) b_{h_{1 T}^{\perp}}^{(S)} \sin \left(\phi_{l}^{h}+\phi_{l}^{S}\right)\right) \\
-2 \frac{2-y}{Q \sqrt{1-y}}\left[S_{\gamma l}^{T} A_{h_{1 T}}^{(S)}\left(\alpha_{h_{1 T}}^{(S)}\left(1-z \alpha_{h_{1 T}}^{(S)}\right) P_{T}^{h 2}-z b_{h_{1 T}}^{(S)}\right) \sin \phi_{l}^{S}\right. \\
+S_{\gamma l}^{L} \frac{A_{h_{1 L}^{\perp}}^{(S)}}{m_{D}}\left(\alpha_{h_{1 L}^{\perp}}^{(S) 2}\left(1-z \alpha_{h_{1 L}^{\perp}}^{(S)}\right) P_{T}^{h 2}+\left(1-2 z \alpha_{h_{1 L}^{\perp}}^{(S)}\right) b_{h_{1 L}^{\perp}}^{(S)}\right) P_{T}^{h} \sin \phi_{l}^{h} \\
+S_{\gamma l}^{T} \frac{A_{h_{1 T}^{\perp}}^{(S)}}{m_{D}^{2}}\left(\frac{1}{2} z\left(\alpha_{h_{1 T}^{\perp}}^{(S) 2} P_{T}^{h 2}+2 b_{h_{1 T}^{\perp}}^{(S)}\right) b_{h_{1 T}^{\perp}}^{(S)} \sin \phi_{l}^{S}\right. \\
\left.\left.\left.-\alpha_{h_{1 T}^{\perp}}^{(S)}\left[\alpha_{h_{1 T}^{\perp}}^{(S) 2}\left(1-z \alpha_{h_{1 T}^{\perp}}^{(S)}\right) P_{T}^{h 2}+\left(2-3 z \alpha_{h_{1 T}^{\perp}}^{(S)}\right) b_{h_{1 T}^{\perp}}^{(S)}\right] P_{T}^{h 2} \cos \left(\phi_{l}^{h}-\phi_{l}^{S}\right) \sin \phi_{l}^{h}\right)\right]\right\} .
\end{gathered}
$$

Here the following notation has been adopted ${ }^{5}$

$$
\begin{aligned}
& B_{J}^{(0, S)}=a_{F}^{(0, S)}+z^{2} a_{J}, \quad \alpha_{J}^{(0, S)}=\frac{z a_{J}}{B_{J}^{(0, S)}}, \quad b_{J}^{(0, S)}=\frac{a_{F}^{(0, S)} a_{J}}{B_{J}^{(0, S)}}, \\
& A_{J}^{(0, S)}=\frac{1}{\pi B_{J}^{(0, S)}} \exp \left(-\frac{P_{T}^{h 2}}{B_{J}^{(0, S)}}\right) d_{J}(x) F^{(0, S)}(z) .
\end{aligned}
$$

One can check that both the $\phi_{l}^{h}$ and $y$ dependence of the parton model result (42)-(45) exactly coincide with that required by the general structure function analysis of section 2.

Integrating (43)-(45) over hadron azimuthal angle one gets

$$
\begin{aligned}
\int_{0}^{2 \pi} d \phi_{l}^{h} C_{U P}= & 2 \pi A_{f_{1}}^{(0)}\left(1+(1-y)^{2}\right) \\
\int_{0}^{2 \pi} d \phi_{l}^{h} C_{D P}= & 2 \pi \lambda y\left(S_{\gamma l}^{L} A_{g_{1 L}}^{(0)}(2-y)-2 S_{\gamma l}^{T} \frac{A_{g_{1 T}}^{(0)}}{m_{D} Q} \sqrt{1-y}\left(\alpha_{g_{1 T}}^{(0) 2} P_{T}^{h 2}+b_{g_{1 T}}^{(0)}\right) \cos \phi_{l}^{S}\right) \\
\int_{0}^{2 \pi} d \phi_{l}^{h} C_{S P}= & 4 \pi \frac{(2-y) \sqrt{1-y}}{Q m_{F}} S_{\gamma l}^{T}\left\{2 A_{h_{1 T}}^{(S)}\left[\alpha_{h_{1 T}}^{(S)}\left(1-z \alpha_{h_{1 T}}^{(S)}\right) P_{T}^{h 2}-z b_{h_{1 T}}^{(S)}\right]\right. \\
& +\frac{A_{h_{1 T}^{\perp}}^{(S)}\left[z\left(\alpha_{h_{1 T}^{\perp}}^{(S) 2} P_{T}^{h 2}+2 b_{h_{1 T}^{\perp}}^{(S)}\right) b_{h_{1 T}^{\perp}}^{(S)}\right.}{m_{D}^{2}}\left[\begin{array}{l}
(S) \\
\end{array}\right. \\
& \left.\left.-\alpha_{h_{1 T}^{\perp}}^{(S)}\left(\alpha_{h_{1 T}^{\perp}}^{(S) 2}\left(1-z \alpha_{h_{1 T}^{\perp}}^{(S)}\right) P_{T}^{h 2}+\left(2-3 z \alpha_{h_{1 T}^{\perp}}^{(S)}\right) b_{h_{1 T}^{\perp}}^{(S)}\right) P_{T}^{h 2}\right]\right\} \sin \phi_{l}^{S} .
\end{aligned}
$$

As is clear from the last equation, even when integrated over hadron azimuthal angle, the SIDIS cross section can still have a single target spin asymmetry. Further integration over hadron transverse momentum gives

$$
\int d^{2} P_{T}^{h} C_{U P}=\left(1+(1-y)^{2}\right) f_{1}(x) F^{(0)}(z),
$$

\footnotetext{
${ }^{5}$ To simplify notation the index $q$ has been suppressed where possible. Obviously, all quantities related with DF (FF) depend on quark flavor $q$ (and final hadron type $h$ ).
} 


$$
\begin{aligned}
\int d^{2} P_{T}^{h} C_{D P}= & \lambda y\left[S_{\gamma l}^{L}(2-y) g_{1 L}(x)-2 S_{\gamma l}^{T} \sqrt{1-y} \frac{a_{g_{1 T}}}{m_{D} Q} g_{1 T}(x) \cos \phi_{l}^{S}\right] F^{(0)}(z), \\
\int d^{2} P_{T}^{h} C_{S P}= & 4 \frac{(2-y) \sqrt{1-y}}{Q m_{F}} S_{\gamma l}^{T}\left\{h_{1 T}(x)\left[\alpha_{h_{1 T}}^{(S)}\left(1-z \alpha_{h_{1 T}}^{(S)}\right) B_{h_{1 T}}^{(S)}-z b_{h_{1 T}}^{(S)}\right]\right. \\
& \left.+\frac{a_{h_{1 T}^{\perp}}}{m_{D}^{2}} h_{1 T}^{\perp}(x)\left[\alpha_{h_{1 T}^{\perp}}^{(S)}\left(1-z \alpha_{h_{1 T}^{\perp}}^{(S)}\right) B_{h_{1 T}^{\perp}}^{(S)}-z b_{h_{1 T}^{\perp}}^{(S)}\right]\right\} F^{(S)}(z) \sin \phi_{l}^{S} .
\end{aligned}
$$

Using definitions (46), one can check that expressions in the square brackets on the rhs of the last equation are equal to zero. Thus, in the quark-parton model the single-spin asymmetry disappears already after the $\vec{P}_{T}^{h}$-integration, and sum rule $(22)$ holds.

Finally, let us multiply the two first equations in (48) by $z$, integrate over $z$ and sum over final hadron types. Using the well-known momentum sum rule,

$$
\sum_{h} \int d z z F^{(0)}(z)=1
$$

the following relations between polarized DIS structure functions and quark DF's are established:

$$
\begin{aligned}
& F_{1}^{D I S}\left(x, Q^{2}\right)=\frac{1}{2} \sum_{q} e_{q}^{2} f_{1}(x) \\
& g_{1}^{D I S}\left(x, Q^{2}\right)=\frac{1}{2} \sum_{q} e_{q}^{2} g_{1 L}(x) \\
& g_{2}^{D I S}\left(x, Q^{2}\right)=\frac{1}{2} \sum_{q} e_{q}^{2}\left(\frac{a_{g_{1 T}}}{2 M m_{D} x} g_{1 T}(x)-g_{1 L}(x)\right) .
\end{aligned}
$$

The last equation in (50) shows that both the "longitudinal", $g_{1 L}\left(x, k_{T}^{2}\right)$, and "transverse", $g_{1 T}\left(x, k_{T}^{2}\right)$, parts of the quark longitudinal-spin distribution give a twist-two contribution to the $g_{2}^{D I S}\left(x, Q^{2}\right)$. This result with quark mass and higher-twist corrections has been found by Tangerman and Mulders (see eq. 2.29 in Ref. [14] and the discussion therein).

\section{Discussion}

In section 4 the cross section for the production of a single unpolarized hadron was calculated. However, with a parton model expression (36) for the polarization density matrix of the final quark, one can also calculate the cross sections for the production of polarized self-analyzing baryons or multi-hadron states (double-particle Collins effect, handedness, ...).

To investigate transverse spin distributions, it seems more convenient to use an unpolarized lepton beam and a polarized target because, in this case, six of the structure functions in (18) do not contribute to the cross section, i.e., $C_{D P}=0$ in the parton model.

I would like to make several remarks here.

In the parton model calculations of section 4 , terms $\sim 1 / Q$ have been kept. In general to get self-consistent results to this order in $1 / Q$ one has to take into account also firstorder QCD radiative corrections and twist-three DF's and FF's. However, as one can 
conclude from the EMC data analysis [12], the most important contribution to azimuthal dependence comes from kinematical effect of the intrinsic transverse momentum (eq. 43), as calculated by Cahn [11].

In some articles concerning polarized DIS and SIDIS only $f_{1}(x), g_{1}(x)$ and $h_{1}(x)$ are considered. They are related to the DF's used in section 3 by $\vec{k}_{T^{-}}$integration

$$
\begin{aligned}
& f_{1}(x)=\int d^{2} k_{T} f_{1}\left(x, k_{T}^{2}\right), \\
& g_{1}(x)=\int d^{2} k_{T} g_{1 L}\left(x, k_{T}^{2}\right)=g_{1 L}(x), \\
& h_{1}(x)=\int d^{2} k_{T}\left[h_{1 T}\left(x, k_{T}^{2}\right)+\frac{k_{T}^{2}}{2 m_{D}^{2}} h_{1 T}^{\perp}\left(x, k_{T}^{2}\right)\right]=h_{1 T}(x)+\frac{a_{h_{1 T}^{\perp}}}{2 m_{D}^{2}} h_{1 T}^{\perp}(x) .
\end{aligned}
$$

The DF's $g_{1 T}(x)$ and $h_{1 L}(x)$ do not give contributions to $g_{1}(x)$ and $h_{1}(x)$.

However, the nonperturbative effects of intrinsic transverse momentum play an important role for polarized SIDIS when dependence on azimuthal angle of the produced hadron is considered. First of all, even in the zeroth approximation in $1 / Q$, all six twist-two DF's contribute to the cross section in this case and the dependence on the produced hadron azimuthal angle, though explicitly calculable, is rather complicated.

The quark-parton model calculations of Ref. [3], [6] and [7] has been performed, neglecting the effects of quark intrinsic transverse momentum. This corresponds to a $\delta(\vec{k})$ distribution for intrinsic $k_{T}$ or, in our notation, to $a_{J}=0$ and so $\alpha_{J}^{(0, S)}=0$ and $b_{J}^{(0, S)}=0$. This rather arbitrary assumption is not true in the case of $f_{1}\left(x, k_{T}^{2}\right)$. Experiments indicate that $a_{f_{1}}$ can reach a value of $\approx 0.4(G e V / c)^{2}$ (see [12] and [20]).

Let us consider (44)-(47) in the zeroth approximation in $1 / Q$. In this approximation $\phi_{c o l}=\phi_{l}^{h}+\phi_{l}^{S}-\pi$. In the original paper by Collins [3] $a_{J}=0$ was assumed and only the first term on the rhs of eq. (45) was obtained. But, as one can see from (42)-(45), the azimuthal dependence of the polarized SIDIS cross section exhibits more complicated behavior already at zeroth order in $1 / Q$ when intrinsic $k_{T}$ effects are taken into account.

I would like to stress here that, in contrast to parton model result for the azimuthal asymmetry in unpolarized SIDIS [11], in the case of polarized SIDIS, the azimuthal dependence appears at leading order in $Q^{2}$. Thus, in polarized SIDIS there are not only $\sim 1 / Q$ effects of kinematical origin (as in the unpolarized case) but also leading ones coming from the azimuthal dependence of the initial quark distribution and/or polarized fragmentation. Suppose for example that the polarized part of the FF is equal to zero. Then $C_{S P}=0$, but one can still have a leading-order azimuthal asymmetry in the second term of the rhs of (44) due to the nonzero longitudinal spin of the initial quark in the transversely-polarized nucleon (if $g_{1 T} \neq 0$ ).

\subsection{Examples of the target spin asymmetries.}

Suppose that the SIDIS cross section is measured for different beam helicities and target polarizations. Then, using the specific forms of the $\phi_{l}^{h}, \phi_{l}^{S}, P_{T}^{2}$ and $y$ dependence of the different terms in (42)-(45) one can separate the contribution of the different DF's and FF's.

For example, consider the case of a longitudinally-polarized (in the laboratory frame) lepton and target. The target longitudinal polarization asymmetry with fixed lepton beam 
helicity, defined as 0

$$
\mathcal{A}_{L}=\frac{d \sigma^{\rightarrow}-d \sigma^{\leftarrow}}{d \sigma^{\rightarrow}+d \sigma^{\leftarrow}}
$$

to zeroth order in $1 / Q$ is given by

$$
\mathcal{A}_{L}=\frac{\mathcal{C}_{g_{1 L}}^{(0)}+\mathcal{C}_{h_{1 L}^{\perp}}^{(S)}}{\mathcal{C}_{f_{1}}^{(0)}},
$$

where the contribution of different DF's and FF's are:

$$
\begin{aligned}
& \mathcal{C}_{f_{1}}^{(0)}=\left[1+(1-y)^{2}\right] \sum_{q} e_{q}^{2} A_{f_{1}}^{(0)}, \\
& \mathcal{C}_{g_{1 L}}^{(0)}=\lambda y(2-y) \sum_{q} e_{q}^{2} A_{g_{1 L}}^{(0)}, \\
& \mathcal{C}_{h_{1 L}^{\perp}}^{(S)}=-2(1-y) \sin 2 \phi_{l}^{h} \frac{P_{T}^{h 2}}{m_{D} m_{F}} \sum_{q} e_{q}^{2} \alpha_{h_{1 L}^{\perp}}^{(S)}\left(1-\alpha_{h_{1 L}^{\perp}}^{(S)}\right) A_{h_{1 L}^{\perp}}^{(S)} .
\end{aligned}
$$

As is clear from(54), one can separate the contributions of $\mathcal{C}_{g_{1 L}}^{(0)}$ and $\mathcal{C}_{h_{1 L}^{\perp}}^{(S)}$ by measuring the target longitudinal-spin asymmetry for different values of $\phi_{l}^{h}, P_{T}^{h}$ and $y$. If the experiment shows that $\mathcal{C}_{h_{1 L}^{\perp}}^{(S)} \neq 0$, then one can conclude that both the twist-two DF, $h_{1 L}^{\perp}$, and FF, $F^{(S)}$, are nonzero. Thus, in principle, it is possible to investigate the Collins effect (a spin dependent $\mathrm{FF}$ ) with a longitudinally polarized beam and target by analyzing existing SMC semi-inclusive data in different bins of $\phi_{l}^{h}$, as was done without such binning in [8].

Analogously one can consider the target transverse polarization asymmetry with a fixed helicity of the lepton beam, defined by

$$
\mathcal{A}_{T}=\frac{d \sigma^{\uparrow}-d \sigma^{\downarrow}}{d \sigma^{\uparrow}+d \sigma^{\downarrow}} .
$$

In the zeroth order on $1 / Q$

$$
\mathcal{A}_{T}=\frac{\mathcal{C}_{g_{1 T}}^{(0)}+\mathcal{C}_{h_{1 T}}^{(S)}+\mathcal{C}_{h_{1 T}^{\perp}}^{(S)}}{\mathcal{C}_{f_{1}}^{(0)}}
$$

with

$$
\begin{aligned}
& \mathcal{C}_{g_{1 T}}^{(0)}= \lambda y(2-y) \frac{P_{T}^{h}}{m_{D}} \cos \left(\phi_{l}^{h}-\phi_{l}^{S}\right) \sum_{q} e_{q}^{2} \alpha_{g_{1 T}}^{(0)} A_{g_{1 T}}^{(0)}, \\
& \mathcal{C}_{h_{1 T}}^{(S)}=-2(1-y) \frac{P_{T}^{h}}{m_{F}} \sin \left(\phi_{l}^{h}+\phi_{l}^{S}\right) \sum_{q} e_{q}^{2}\left(1-z \alpha_{h_{1 T}}^{(S)}\right) A_{h_{1 T}}^{(S)}, \\
& \mathcal{C}_{h_{1 T}^{\perp}}^{(S)}=-2(1-y) \frac{P_{T}^{h}}{m_{F} m_{D}^{2}} \sum_{q} e_{q}^{2} A_{h_{1 T}^{\perp}}^{(S)}\left[\left(\frac{1}{2}-z \alpha_{h_{1 T}^{\perp}}^{(S)}\right) b_{h_{1 T}^{\perp}}^{(S)} \sin \left(\phi_{l}^{h}+\phi_{l}^{S}\right)\right. \\
&\left.\quad+\alpha_{h_{1 T}^{\perp}}^{(S)}\left(1-z \alpha_{h_{1 T}^{\perp}}^{(S)}\right) P_{T}^{h 2} \cos \left(\phi_{l}^{h}-\phi_{l}^{S}\right) \sin 2 \phi_{l}^{h}\right] .
\end{aligned}
$$

\footnotetext{
${ }^{6}$ For simplicity $100 \%$ target polarization is considered.
} 
The contributions of $\mathcal{C}_{h_{1 T}}^{(S)}$ and $\mathcal{C}_{h_{1 T}^{\perp}}^{(S)}$ arising from spin-dependent part of FF can be suppressed if $\mathcal{A}_{T}$ is measured in small bins around $\phi_{l}^{h} \approx \phi_{l}^{S} \approx 0$. In these bins the asymmetry is given by

$$
\mathcal{A}_{T} \approx \frac{\lambda y(2-y) P_{T}^{h} \sum_{q} e_{q}^{2} \alpha_{g_{1 T}}^{(0)} A_{g_{1 T}}^{(0)}}{\left[1+(1-y)^{2}\right] m_{D} \sum_{q} e_{q}^{2} A_{f_{1}}^{(0)}} .
$$

In this way it seems feasible to investigate the twist-two DF, $g_{1 T}$, already with existing SMC semi-inclusive data 21] [1 on a transversely polarized nucleon.

As it is clear from the preceding examples, with a sufficient amount of experimental data it is possible to separate the contributions of the different DF's and FF's by considering appropriate asymmetries in different bins of $\phi_{l}^{h}$ and $\phi_{l}^{S}$ or using the Fourier-analysis method. It is also possible to perform a flavor analysis, as proposed in Ref. [5]-[7], by measuring SIDIS asymmetries on proton and deuteron targets for different types of final hadron.

Unfortunately, there are no measurements or theoretical calculations of the "new" DF's $g_{1 T}, h_{1 T}^{\perp}$ and $h_{1 L}^{\perp}$ or the polarized part of the FF's, and numerical estimation of different asymmetries in polarized SIDIS is now impossible.

\section{Conclusions}

In this paper polarized SIDIS has been considered in the quark-parton model with a nonzero intrinsic $k_{T}$, taking into account all six leading-twist DF's and the Collins effect in the FF's and keeping kinematical corrections of order $\sim 1 / Q$. It is shown that already at zeroth order in $1 / Q$ the effects of intrinsic $k_{T}$ are not negligible: all six twist-two DF's with the polarized part of the FF's give contributions to the azimuthal dependence of the cross section. A study of appropriate asymmetries in polarized SIDIS will allow an investigation of the different DF's and FF's at twist-two. It is possible to start this type of analysis already with existing SMC semi-inclusive data.

It was also noticed that a single target-spin asymmetry (with an unpolarized lepton beam) can exist in the SIDIS cross section when integrated over final hadron azimuthal angle. In the quark-parton model this asymmetry is of order $1 / Q$, and disappears after $P_{T}^{h 2}$ integration.

New polarized SIDIS experiments like thoss proposed by the HELP and HERMES collaborations will certainly be very instructive for the investigation of the new DF's and FF's.

\section{Acknowledgements}

I wish to thank G. Altarelli, X. Artru, E. Berger, L.Dick, A. Efremov, P.G. Ratcliffe, G. Veneziano and B. Vuaridel for useful discussions.

This work was supported in part by Professor Louis Dick and Geneva University.

\footnotetext{
${ }^{7}$ In the Ref. [21 only inclusive data was analyzed.
} 


\section{References}

[1] J.P. Ralston and D.E. Soper,Nucl.Phys. B152, 109,(1979).

[2] R.L. Jaffe and Xiangdong Ji, Nucl.Phys. B375, 527 (1992).

[3] J. Collins Nucl.Phys. B369, 161 (1993); Nucl.Phys. B375, 527 (1992).

[4] X.Artru, in Proceeding of the Polarized Collider Workshop, University Park, Eds. J. Collins, S.F. Heppelman, R.W. Robinett, AIP 223, 176 (1990) and X. Artru and M. Mekhfi, Z. Phys. C45, 669 (1990); Nucl. Phys A532, 351 (1991).

[5] The HELP collaboration "A proposal for an Internal Jet-Target Experiment at LEP", CERN/LEPC 93-14, LEPC/P7; CERN/LEPC 94-1, LEPC/P7 Add. 1, and refs. therein.

[6] F.E. Close and R.G. Milner, Phys.Rev. D44, 3691 (1991).

[7] L.L. Frankfurt et al., Phys.Lett. B230, 141 (1989).

[8] W. Wislicki, (SMC), Polarization of Valence and Sea Quarks in the Proton., Talk presented at XXIXth RENCONTRES DE MORIOND QCD and HIGH ENERGY HADRONIC INTERACTIONS, Meribel, France,22nd March 1994, hep-ex/9405012.

[9] The HERMES collaboration "Technical Design Report", DESY-PRC 93/06, MPIHV20-1993.

[10] R.L. Jaffe and Xiangdong Ji, Phys. Rev. Lett. 71,2547 (1993).

[11] R.N. Cahn, Phys.Lett. 78B, 269 (1978), Phys.Rev. D40, 3107 (1989).,

[12] M. Arneodo et al., (EMC), Z. Phys.C 34,277,(1987).

[13] R.D. Tangerman and P.J. Mulders, NIKHEF-94-P1, hep-ph/9403227

[14] R.D. Tangerman and P.J. Mulders, NIKHEF-94-P7, hep-ph/9408305

[15] N. Dombey, Rev.Mod.Phys. 41,236,(1969).

[16] M. Gourdin, Nucl. Phys. B49, 501 (1972).

[17] S. Boffi, C. Giusti and F.D. Pacati, Phys. Rep. 226, 1 (1993).

[18] R.L. Jaffe, Comments Nucl. Part. Phys. 19, 239 (1990).

[19] Landau and Lifshitz, Course of Theoretical Physics. Vol. 4 2nd Edition, Pergamon Press.

[20] J. Chay, S.D. Ellis and W.J. Stirling, Phys.Rev. D45, 46 (1992).

[21] D. Adams et al., (SMC), Phys.Lett. B 336, 125 (1994). 